\title{
Shared Decision Making and Motivational Interviewing: Achieving Patient-Centered Care Across the Spectrum of Health Care Problems
}

Glyn Elwyn, $M D, P b D^{1}$

Cbristine Deblendorf, MD, MAS

Ronald M. Epstein, $M D^{3}$

Katy Marrin, MSc ${ }^{4}$

James White, $\mathrm{PbD}^{4}$

Dominick L. Frosch, $\mathrm{PbD}^{5,6,7}$

'The Dartmouth Center for Health Care Delivery Science, Hanover, New Hampshire

${ }^{2}$ Departments of Family \& Community Medicine, Obstetrics, Gynecology \& Reproductive Sciences, and Epidemiology \& Biostatistics, UCSF, San Francisco, California

${ }^{3}$ Department of Family Medicine, University of Rochester Medical Center, Rochester, New York

${ }^{4}$ Cochrane Institute of Primary Care and Public Health, Cardiff University, Heath Park, United Kingdom

${ }^{5}$ Gordon and Betty Moore Foundation, Palo Alto, California

${ }^{6}$ Department of Health Services Research, Palo Alto Medical Foundation Research Institute, Palo Alto, California

${ }^{7}$ Department of Medicine, University of California, Los Angeles, Los Angeles, California

Conflicts of interest: Drs Elwyn and Frosch bave received grants and traveling and speaking honoraria from the Informed Medical Decisions Foundation, Boston. Dr Elwyn consults for Emmi Solutions, USA, a producer of patient decision support. Dr Epstein bas received grants from the Informed Medical Decisions Foundation, Boston. Dr Rollnick provides workshops in motivational interviewing. Dr White is funded by DECIPHer, a UKCRC Public Health Research Centre of Excellence.

\section{CORRESPONDING AUTHOR}

Glyn Elwyn, PhD

The Dartmouth Center for Health Care Delivery Science

Dartmouth-Hitchcock Medical Center

One Medical Center Dr

Lebanon, NH 03756

glynelwyn@gmail.com

\begin{abstract}
Patient-centered care requires different approaches depending on the clinical situation. Motivational interviewing and shared decision making provide practical and well-described methods to accomplish patient-centered care in the context of situations where medical evidence supports specific behavior changes and the most appropriate action is dependent on the patient's preferences. Many clinical consultations may require elements of both approaches, however. This article describes these 2 approaches-one to address ambivalence to medically indicated behavior change and the other to support patients in making health care decisions in cases where there is more than one reasonable option-and discusses how clinicians can draw on these approaches alone and in combination to achieve patient-centered care across the range of health care problems.
\end{abstract}

Ann Fam Med 2014;270-275. doi: 10.1370/afm.1615.

\section{INTRODUCTION}

$\mathrm{D}$ uring the past several decades, patients' values, preferences, and experiences have been given increasing emphasis in clinical interactions in an effort to promote patient-centered care. ${ }^{1}$ Patient-centered care has been found to be associated with improved patient outcomes, including improved self-management, patient satisfaction, and medication adherence, and some studies have found evidence for improved clinical outcomes. ${ }^{2,3}$ Data from surveys and qualitative and observational research indicate that clinicians often do not take patients' perspectives into account; rather, clinicians often promote or recommend specific treatments rather than consider patients' preferences during the decision-making process. ${ }^{4-7}$

Clinicians are commonly challenged by the diversity of situations that arise in practice when they attempt to implement patient-centered care. For example, providing patient-centered care for a patient at the end of life is very different from counseling a patient with a long-term health condition or providing advice about preventative care. Each situation has different psychosocial, cultural, and medical implications. A key factor is the degree to which a clinical situation has acceptable alternative courses of action, ie, situations of equipoise, ${ }_{1}^{8}$ or whether there is clear evidence for a preferred course of action. For the patient electing to have a mastectomy or lumpectomy in early breast cancer, equipoise exists about the long-term outcomes. Evidence for a preferred course of action is found for the overweight smoker with diabetes who is encouraged to consider quitting.

Clearly, different situations require different communication approaches, and patient-centered approaches for each of these situations have been delineated during the last few decades. We wish to focus this article on 2 specific methods, namely, shared decision making and motivational interviewing. As researchers and practitioners, we also wish to share our experience with both. In this article, we provide guidance for how to apply 
patient-centered approaches across a range of clinical problems. In doing so, we explore the definitions of shared decision making and motivational interviewing and summarize the evidence on their use. We also consider the overlap between the 2 approaches and discuss how practitioners can flexibly combine them to improve their patient-centered practice.

\section{MOTIVATIONAL INTERVIEWING AND SHARED DECISION MAKING}

There has been increasing interest in the concept of shared decision making, in which the clinicians' role is to help patients understand what the reasonable options are, then elicit, inform, and integrate patients informed preferences as they relate to the available options. Motivational interviewing has also received attention as a patient-centered approach to counseling for guiding behavior change, usually when a patient feels ambivalent, eg, about lifestyle choices or adherence to medication. ${ }^{9,10}$ A motivational interviewing approach enables clinicians to have a goal for counseling while acknowledging and exploring variation in individuals' commitment to and interest in changing their behavior.

Clinicians must be able to identify situations where these methods are most appropriate and recognize that sometimes both methods may be required. Doing so can introduce complexity in providing patient-centered care, and many clinical problems do not fit neatly into one or another category. For example, many problems can have one more-effective option but still have multiple acceptable options given the patient's preferences. A good example is with treatment of hypertension, the clinician may wish to promote a specific medication but would be willing to prescribe a different, slightly less effective medication if it resulted in the patient being more likely to adhere to the medication regimen. In addition, many problems involve a range of trade-offs. For example, a clinician may wish to encourage an obese patient to lose weight and draw upon motivational interviewing to elicit a commitment to weight loss. Once achieved, however, it may be most appropriate to use shared decision making to determine the best method for the patient to lose weight, eg, diet, exercise, or medical interventions.

These complexities make patientcentered care difficult for practitioners, and we think the lack of clarity about how to communicate appropriately in these differing situations contributes to clinicians' documented failure to use a patientcentered approach.

\section{Shared Decision Making}

Shared decision making is a method "where clinicians and patients make decisions together using the best available evidence, where patients are encouraged to consider available screening, treatment, or management options and the likely benefits and harms of each." ${ }^{\prime 11}$ In this approach, the clinicians' role is to help patients become well-informed, help them develop their personal preferences for available options, and provide professional guidance where appropriate. Most existing research on shared decision making has considered episodic one-time decisions, such as whether to have surgery. Shared decision making can also have a much broader scope and be applied to all situations where competing options exist or approaches need prioritization.

Figure 1 shows a simplified way of thinking about shared decision making in clinical practice. ${ }^{12}$ The figure assumes that the practitioner has achieved the first step of building a constructive relationship and that a decision is needed. Three steps are then shown:

1. Explain the need to consider alternatives as a team (team talk)

2. Describe the alternatives in more detail (option talk); use decision support tools when possible and appropriate

3. Help patients explore and form their personal preferences (decision talk)

\section{Motivational Interviewing}

Motivational interviewing is focused on helping patients identify and resolve ambivalence about changing their behavior, typically by exploring their personal perspectives as well as perceived barriers. Motivational interviewing is most often applied in situations that usually require some degree of behavior change about which a patient feels ambivalent, eg, about lifestyle choices or adherence to medications. Originally developed for dealing with drug and alcohol addiction, the scope of motivational interviewing has widened to include how best to motivate behavior change across many domains. ${ }^{14}$ Patient (or client) centeredness is a
Figure 1. Shared decision making.

Initial
preferences




\section{Figure 2. Motivational interviewing.}

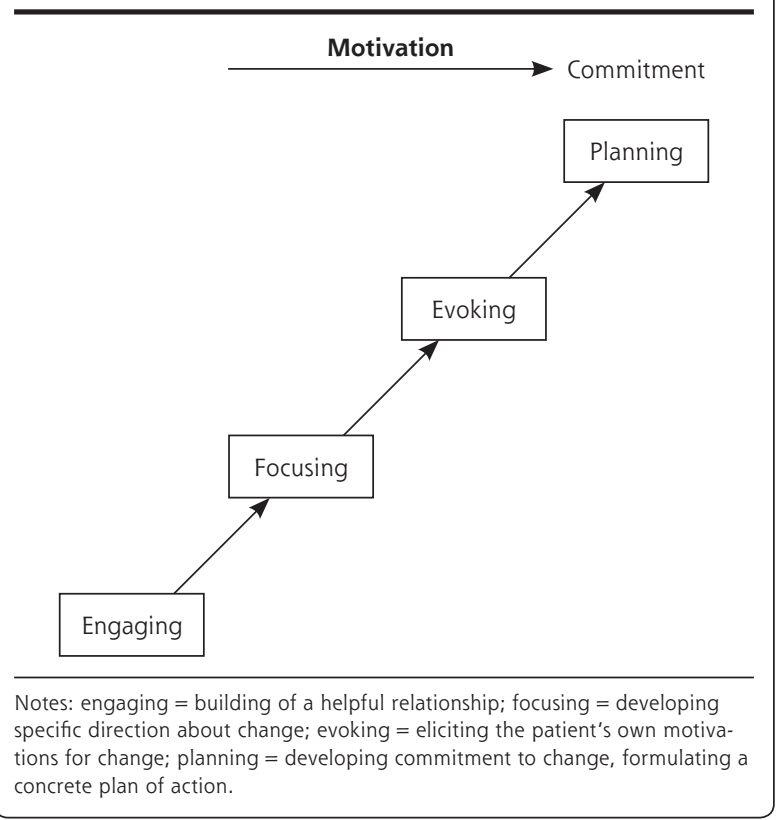

core principle: motivational interviewing recognizes that making behavioral changes is difficult and that telling or persuading people to change will often meet with resistance. Instead of viewing resistance as a problem (or failure), motivational interviewing approaches resistance as ambivalence that should be "explored and resolved"14 and in so doing elicits and encourages patient's own motives to change.

Motivational interviewing involves 4 overlapping and additive steps: (1) engaging, (2) focusing, (3) evoking, and (4) planning (Figure 2). Engaging refers to building a helpful working relationship and is a prerequisite for focusing, a process during which a specific direction about change is developed and maintained in the conversation. Evoking involves eliciting the patient's own motivations for change; their ideas and feeling are recognized, elicited, explored, and reinforced. Planning encompasses both developing commitment to change and formulating a concrete plan of action: it is a conversation about action, eliciting the patient's own solutions and continuing to strengthen talk about change as a plan emerges.

Both motivational interviewing and shared decision making are patient-centered methods that promote the ethical imperative of respecting autonomy, and both have been associated with improved patient outcomes. The strongest evidence for shared decision making comes from the use of decision support tools. A systematic review and meta-analysis of 86 trials suggests consistent improvement in patients' knowledge and more accurate perceptions of risk, leading to increased confidence in decisions. ${ }^{15}$ In addition, in some trials patients have decided not to undergo elective surgery after becoming better informed..$^{15}$ Other trials have found improvements in patients' ability to self-manage long-term conditions and adherence to medication. ${ }^{16}$ Most research, though, has pertained to one-time dichotomous decisions; more work is in progress about how shared decision making is relevant to ongoing decisions for long-term conditions. ${ }^{17}$

For motivational interviewing, there is evidence for efficacy in treating addictions and mixed evidence for efficacy in improving health outcomes of patients with diabetes, asthma, high blood pressure, and heart disease. ${ }^{18-21}$ Most studies have found positive results on lifestyle change outcomes and on psychological outcomes, although some trials have been described as having methodological limitations. ${ }^{22}$ Yet, the principles and methods of motivational interviewing are highly valued by practitioners frustrated with the ineffectiveness of the traditional prescriptive advice giving. ${ }^{20,23}$

\section{Integrating Shared Decision Making and Motivational Interviewing}

Both shared decision making and motivational interviewing focus on engaging patients to explore their views and opinions, including options for treatment or management approaches from the patients' perspective. Although traditionally these methods have been applicable in distinct and nonoverlapping situations, practitioners may benefit from drawing on both approaches to maintain a patient-centered orientation in real-world clinical situations.

Figure 3 illustrates the contrasting goals and contexts of shared decision making and motivational interviewing, as well as their overlap and the interrelationships between the associated principles and skills. As discussed, motivational interviewing is focused on supporting change away from risky behavior toward a specific evidence-based behavior change goal, such as toward a health-enhancing behavior (reducing smoking or excessive drinking), or toward a physical state that conveys less risk to health (managing blood glucose levels, maintaining a body mass index of 25 or less). In contrast, shared decision making has been considered relevant when weighing reasonable options to make a decision on treatment. These processes share common components and can and should be integrated to achieve patient-centered goals.

Motivational interviewing and shared decision making respect autonomy and build relationships based on respect for and curiosity about the patient as a person. Both rely on fundamental communication skills-developing trust, understanding, empathy, and patient enablement facilitate decision making and behavior change. 


\section{Figure 3. The relationship of shared decision making and motivational interviewing.}

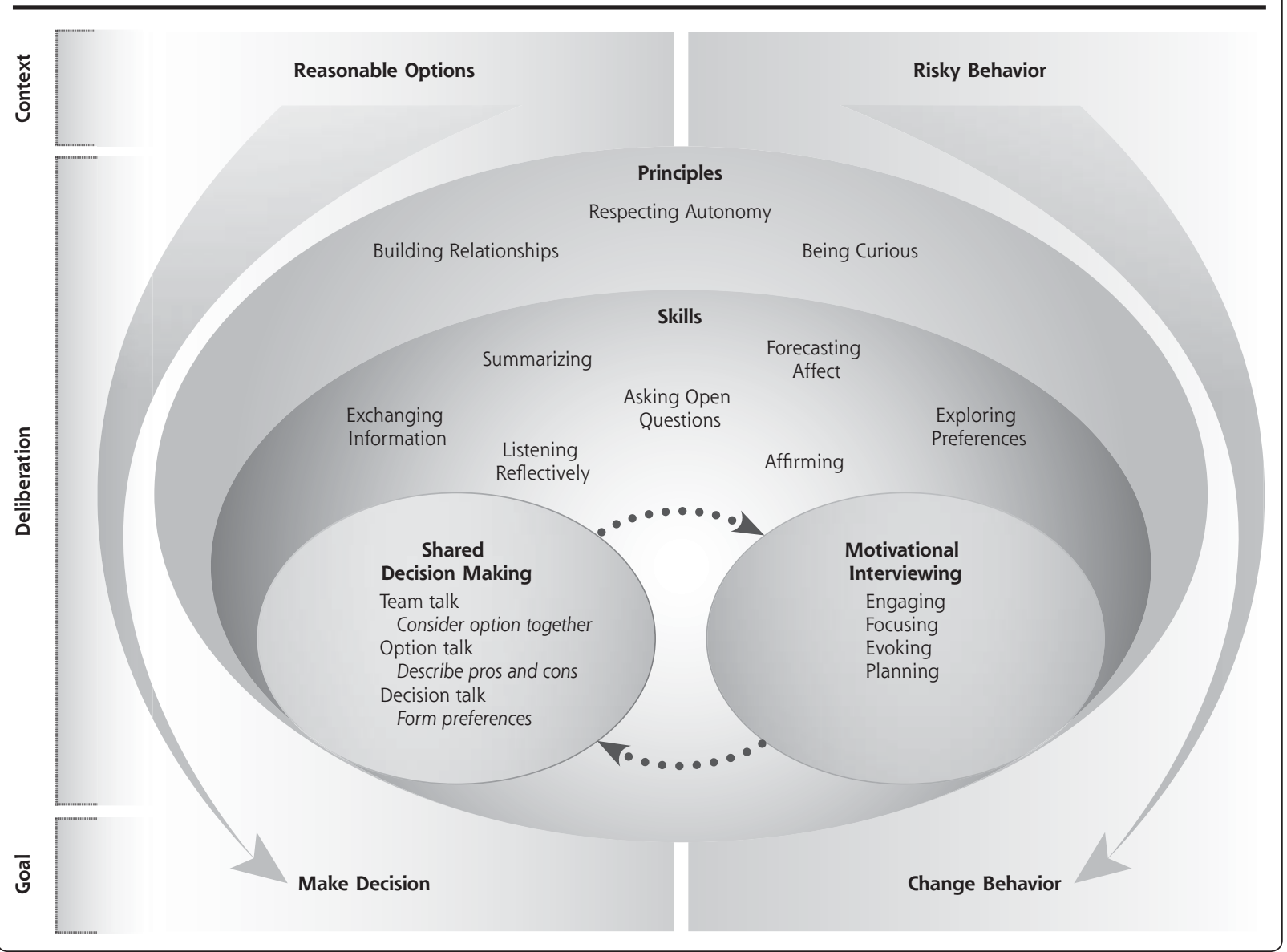

The aims of both methods are accomplished through exchanging information, reflective listening, and responding to emotions. Depending on the goal, practitioners use these fundamental skills to lesser or greater extents. Motivational interviewing addresses ambivalence to change; the interviewer seeks to explore and understand the patient's reasons to change before setting out a plan of action. What, for instance, is the benefit of smoking to the smoker? Why might that person want to stop smoking? How important is it to stop or to continue smoking? In contrast, shared decision making strives to clarify treatment options and help a patient to actively consider those options before supporting a journey toward informed, well-considered preferences and confident decisions. As shown in Figure 3, these complementary processes can be integrated in providing counseling for such long-term conditions as diabetes, as well as for behavioral changes, such as weight loss.

\section{A Practical Example}

To illustrate clinical situations in which integration of these methods can be appropriate, we consider Bill, who is 55 years old and has problems with his type 2 diabetes mellitus (Figure 4). He lives with his wife and works long hours as a factory security guard.

In this example, the medical literature provides ample evidence that Bill's health outcomes will be improved if his diabetes were better controlled. His practitioner wants to promote improved blood glucose control, and Bill has a range of options. To improve his risk profile, he could take more medication (or use insulin), as well as lose weight and exercise. It is also possible that by losing weight and by exercising more, it may be unnecessary for Bill to take more medication. Bill, however, is ambivalent about making any changes.

Figure 4 illustrates how shared decision making and motivational interviewing can used to help Bill choose how to manage his problems. The first key task of the patient-centered practitioner is to establish rapport and trust with Bill, including communicating curiosity and respect for his views and priorities. In addition, it is important that the practitioner and Bill have a common understanding of the issue to be addressed during the visit. While emphasizing 
that improved blood pressure and glucose levels will improve his long-term health outcomes, the practitioner can draw on motivational interviewing to help Bill explore his ambivalence about making changes. The practitioner would listen as Bill considers what is important and draw out Bill's reasons for making or not making changes. Motivational interviewing would help Bill explore his ambivalence to change, focus on a goal that he might mention, and then help him build confidence in achieving that goal while the practitioner attempts to avoid blame or guilt.

When discussing his commitment to change, Bill will need to consider whether he finds any possible options to be acceptable, and if so, which are best given his circumstances and priorities. The practitioner's use of shared decision making helps to compare options by discussing the pros and cons of potential treatments and listening carefully to concerns as Bill forms his preferences informed by new information.

Motivational interviewing and shared decision making can be applied sequentially so that motivating patients to change is followed by decision making to help decide on a preferred approach. It is equally possible, and in many cases desirable, to integrate these methods as an ongoing process. Clinicians may, for example, provide information about options before eliciting patients' preferences-part of a shared decision-making approach — then guide their counsel- ing based on the degree of acceptable behavior change given the patients' level of ambivalence.

In Bill's case, using an integrated approach by drawing on both motivational interviewing and shared decision making can help address Bill's ambivalence toward change and identify the most realistic option for addressing his chronic health conditions, as well as help develop a plan for change. Ultimately, Bill decides to tackle his adherence to medication, work with his wife regarding his need for dietary changes, and attempt to exercise more at home. He will continue to consider whether and when he might address the more difficult lifestyle changes that would reduce the need for more intensive treatment.

\section{CONCLUSIONS}

Providing patient-centered care consistently in clinical practice requires practitioners who are able to recognize that different clinical situations require different approaches and are skilled enough to adapt and, where needed, integrate methods. When patients face tough treatment decisions, shared decision making alone is appropriate. Where clinicians perceive a need to change behavior to improve health outcomes, motivational interviewing could be used. These 2 methods can be integrated when behavior change and choosing between competing options are relevant. Identifying

\section{Figure 4. Bill is overweight and having trouble with his diabetes.}

Bill is overweight (body mass index $=35$ ) and has persistent high blood pressure and high glycosylated hemoglobin levels, even though he has received prescriptions for high doses of medication. He admits to a poor diet, skipping his medication, and no real interest in exercise.

Initial Steps: Practitioner establishes rapport with patient and discusses the context for the decision to be made. In doing so, techniques from both motivational interviewing and shared decision making can be used:

Focusing (motivational interviewing), in which the practitioner directs the conversation toward the need to lower Bill's blood glucose levels Team talk (shared decision making), in which the practitioner reviews the need to consider the different options available for lowering blood glucose levels, creating a constructive dialogue.

\section{Motivational Interviewing}

Evoking

- Avoid persuasion and draw out people's own motives.

Bill's doctor explores what is important to Bill. What reasons might he have to stay as he is or to change? Bill reports that he wishes to lose weight, because he is beginning to notice increased pain in his knees when walking. He also has been bothered by frequent urination that interferes with his ability to do his work as a security guard.

- Listen as they consider change.

His doctor listens, reinforces, and summarizes the change that makes sense to Bill. The physician discovers that Bill does not want to burden on his wife, who does all the cooking. His ability to exercise is limited by his need to care for his elderly mother. His unusual work hours also make it difficult for him to remember to take his pills.

Planning

- Support change planning if ready.

His doctor supports Bill to make a plan for change that might work: bring his wife to the next appointment as the first step, consider types of exercise he can do at home, and use medication reminders on his cell phone.

\section{Shared Decision Making}

Option Talk

- Explain why options need to be considered.

Bill's doctor outlines a number of options and explains why they coexist.

- Provide accurate information about the pros and cons of available options.

Bill could lose weight or increase his exercise levels, preferably do both together, in an effort to bring his body mass index down to less than 30 . He could also take additional oral medication or take his current medication more consistently. Or he could start insulin therapy, but this option is known to lead to further weight gain. Details about these options are shared.

Decision Talk

- Listen and support as people form preferences.

Bill would rather avoid more medication; he already has sideeffects. He is reluctant to consider insulin. His preferences change as he understands more about the trade-offs: he becomes more interested in weight loss when he hears that going on insulin would risk him gaining even more weight. 
the appropriate application of these patient-centered methods, alone and in combination, will assist practitioners in achieving a patient-centered clinical practice.

Finally, we acknowledge the considerable challenge of implementing shared decision making and motivational interviewing into routine practice, let alone integrating them seamlessly as complex patients' needs arise. We believe, however, that we will see little progress in patient-centered care unless these approaches are valued as core elements of good practice; they should be taught, assessed, and integrated into daily practice, then appropriately measured and rewarded. By so doing, we envision a better future for medical practice.

To read or post commentaries in response to this article, see it online at http://www.annfammed.org/content/12/3/270.

Key words: decision making, shared; motivational interviewing; nondirective therapy; concept formation; problem solving

Submitted July 2, 2013; submitted, revised, October 12, 2013; accepted November 19, 2013.

Author contributions: This article is based on research work funded by the Informed Medical Decisions Foundation, Boston. All authors contributed to the drafting. The aim was to illustrate how shared decision making and motivational interviewing could be combined in routine practice. Stephen Rollnick is one of the originators of motivational interviewing; Glyn Elwyn, Christine Dehlendorf, Ronald Epstein, and Dominick Frosch undertake research into shared decision making in the context of patient-centered care. Katy Marrin and James White collaborated in every aspect of this work. The guarantor is Glyn Elwyn.

Funding support: Support from the British Heart Foundation, Cancer Research UK, Economic and Social Research Council (RES-590-28-0005), Medical Research Council, the Welsh Government and the Wellcome Trust (WT087640MA), under the auspices of the UK Clinical Research Collaboration, is gratefully acknowledged. Christine Dehlendorf's contribution was part of a project supported by grant No.K23HD067197 from the Eunice Kennedy Shriver National Institute of Child Health \& Human Development.

Disclaimer: The content is solely the responsibility of the authors and does not necessarily represent the official views of the Eunice Kennedy Shriver National Institute of Child Health \& Human Development or the National Institutes of Health.

Acknowledgments: Vi Luong was the graphic designer who developed Figure 3; and the authors are grateful to William Miller for his contribution to the article. We also thank Phyllis Butow, Micheal Peltenburg, Richard Street, and Gregory Makoul who commented on the research report that was the basis for this article.

\section{References}

1. Institute of Medicine. Crossing the Quality Chasm: A New Health System for the 21st Century. Washington, DC: National Academy Press; 2001

2. Robinson JH, Callister LC, Berry JA, Dearing KA. Patient-centered care and adherence: definitions and applications to improve outcomes. J Am Acad Nurse Pract. 2008;20(12):600-607. http://www. ncbi.nlm.nih.gov/pubmed/19120591. Accessed May 22, 2013.
3. Rathert C, Wyrwich MD, Boren SA. Patient-centered care and outcomes: a systematic review of the literature. Med Care Res Rev. 2012. http://mcr.sagepub.com/content/early/2012/11/18/107755871 2465774.abstract. Accessed Jun 15, 2013.

4. Braddock CH III, Edwards KA, Hasenberg NM, Laidley TL, Levinson W. Informed decision making in outpatient practice: time to get back to basics. JAMA. 1999;282(24):2313-2320.

5. Dellasega C, Añel-Tiangco RM, Gabbay RA. How patients with type 2 diabetes mellitus respond to motivational interviewing. Diabetes Res Clin Pract. 2012;95(1):37-41. http://www.ncbi.nlm.nih.gov/ pubmed/21899911. Accessed Jun 5, 2013.

6. Sheridan NF, Kenealy TW, Kidd JD, et al. Patients' engagement in primary care: powerlessness and compounding jeopardy. A qualitative study. Health Expect. 2012. http://www.ncbi.nlm.nih.gov/ pubmed/23033910. Accessed Jun 15, 2013.

7. Braddock CH III, Fihn SD, Levinson W, Jonsen AR, Pearlman RA. How doctors and patients discuss routine clinical decisions. Informed decision making in the outpatient setting. J Gen Intern Med. 1997;12(6):339-345.

8. Elwyn G, Edwards A, Kinnersley P, Grol R. Shared decision making and the concept of equipoise: the competencies of involving patients in healthcare choices. Br J Gen Pract. 2000;50(460):892-899.

9. Edwards A, Elwyn G. Shared Decision-making. Achieving Evidencebased Patient Choice. 2nd ed. Oxford: Oxford University Press; 2009.

10. Rollnick S, Butler CC, Kinnersley P, Gregory J, Mash B. Motivational interviewing. BMJ. 2010;340:c1900. http://www.ncbi.nlm.nih.gov/ pubmed/20423957. Accessed Oct 22, 2010.

11. Elwyn G, Laitner S, Coulter A, Walker E, Watson P, Thomson R. Implementing shared decision making in the NHS. BMJ. 2010;341:c5146.

12. Elwyn G, Frosch DL, Thomson R, et al. Shared decision making: a model for clinical practice. J Gen Intern Med. 2012;27(10):1361-1367.

13. Mulley AG, Trimble C, Elwyn G. Stop the silent misdiagnosis: patients' preferences matter. BMJ. 2012;345:e6572-e6572.

14. Rollnick S, Miller WR, Butler CC. Motivational Interviewing in Health Care: Helping Patients Change Behavior. New York, NY: Guildford Press; 2007.

15. Stacey D, Bennett CL, Barry MJ, et al. Decision aids for people facing health treatment or screening decisions. Cochrane Database Syst Rev. 2011;(10):CD001431.

16. Joosten EA, DeFuentes-Merillas L, de Weert GHST, Sensky T, van der Staak CP, de Jong CA. Systematic review of the effects of shared decision-making on patient satisfaction, treatment adherence and health status. Psychother Psychosom. 2008;77(4):219-226.

17. Murray E, Charles C, Gafni A. Shared decision-making in primary care: tailoring the Charles et al. model to fit the context of general practice. Patient Educ Couns. 2006;62(2):205-211.

18. Hettema J, Steele J, Miller WR. Motivational interviewing. Annu Rev Clin Psychol. 2005;1:91-111.

19. Rubak S, Sandbaek A, Lauritzen T, Christensen B. Motivational interviewing: a systematic review and meta-analysis. Br J Gen Pract. 2005;55(513):305-312.

20. Söderlund LL, Madson MB, Rubak S, Nilsen P. A systematic review of motivational interviewing training for general health care practitioners. Patient Educ Couns. 2011;84(1):16-26.

21. Welch G. Rose. G., Ernst D. Motivational interviewing and diabetes: what is it, how is it used, and does it work? Diabetes Spectr. 2006;19(1):5-11. http://spectrum.diabetesjournals.org. Accessed Sep 10, 2011.

22. Knight KM, McGowan L, Dickens C, Bundy C. A systematic review of motivational interviewing in physical health care settings. $\mathrm{Br} J$ Health Psychol. 2006;11(Pt 2):319-332.

23. Schoener EP, Madeja CL, Henderson MJ, Ondersma SJ, Janisse JJ. Effects of motivational interviewing training on mental health therapist behavior. Drug Alcohol Depend. 2006;82(3):269-275. 